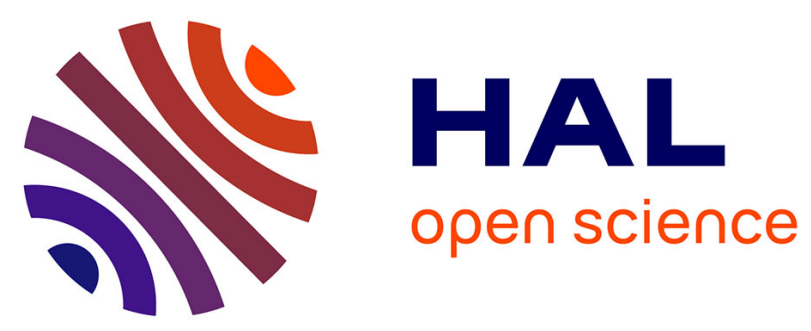

\title{
A propos de la localisation des activités industrielles : le district marshallien
}

\author{
Paul-Marie Romani, Jean-Luc Gaffard
}

\section{To cite this version:}

Paul-Marie Romani, Jean-Luc Gaffard. A propos de la localisation des activités industrielles: le district marshallien. Revue Française d'Economie, 1990, 5 (3), pp.171-185. 10.3406/rfeco.1990.1261 . hal-01009599

\section{HAL Id: hal-01009599 \\ https://hal-sciencespo.archives-ouvertes.fr/hal-01009599}

Submitted on 18 Jun 2014

HAL is a multi-disciplinary open access archive for the deposit and dissemination of scientific research documents, whether they are published or not. The documents may come from teaching and research institutions in France or abroad, or from public or private research centers.
L'archive ouverte pluridisciplinaire HAL, est destinée au dépôt et à la diffusion de documents scientifiques de niveau recherche, publiés ou non, émanant des établissements d'enseignement et de recherche français ou étrangers, des laboratoires publics ou privés.

\section{(ㅇ)(1) $\$$}

Distributed under a Creative Commons Attribution - NonCommercial - NoDerivatives| 4.0 


\title{
Persée
}

\author{
http://www.persee.fr
}

\section{A propos de la localisation des activités industrielles : le district marshallien}

\author{
Paul-Marie Romani;Jean-Luc Gaffard \\ Revue française d'économie, Année 1990, Volume 5, Numéro 3 \\ p. $171-185$
}

Voir l'article en ligne

\section{Avertissement}

L'éditeur du site " PERSEE » - le Ministère de la jeunesse, de l'éducation nationale et de la recherche, Direction de l'enseignement supérieur, Sous-direction des bibliothèques et de la documentation - détient la propriété intellectuelle et les droits d'exploitation. A ce titre il est titulaire des droits d'auteur et du droit sui generis du producteur de bases de données sur ce site conformément à la loi n`98-536 du 1 er juillet 1998 relative aux bases de données.

Les oeuvres reproduites sur le site «PERSEE » sont protégées par les dispositions générales du Code de la propriété intellectuelle.

Droits et devoirs des utilisateurs

Pour un usage strictement privé, la simple reproduction du contenu de ce site est libre.

Pour un usage scientifique ou pédagogique, à des fins de recherches, d'enseignement ou de communication excluant toute exploitation commerciale, la reproduction et la communication au public du contenu de ce site sont autorisées, sous réserve que celles-ci servent d'illustration, ne soient pas substantielles et ne soient pas expressément limitées (plans ou photographies). La mention Le Ministère de la jeunesse, de l'éducation nationale et de la recherche, Direction de l'enseignement supérieur, Sous-direction des bibliothèques et de la documentation sur chaque reproduction tirée du site est obligatoire ainsi que le nom de la revue et- lorsqu'ils sont indiqués - le nom de l'auteur et la référence du document reproduit.

Toute autre reproduction ou communication au public, intégrale ou substantielle du contenu de ce site, par quelque procédé que ce soit, de l'éditeur original de l'oeuvre, de l'auteur et de ses ayants droit.

La reproduction et l'exploitation des photographies et des plans, y compris à des fins commerciales, doivent être autorisés par l'éditeur du site, Le Ministère de la jeunesse, de l'éducation nationale et de la recherche, Direction de l'enseignement supérieur, Sous-direction des bibliothèques et de la documentation (voir http://www.sup.adc.education.fr/bib/ ). La source et les crédits devront toujours être mentionnés. 


\section{J.L. GAFFARD \\ P.M. ROMANI}

\section{A propos de la localisation des activités industrielles : le district marshallien}

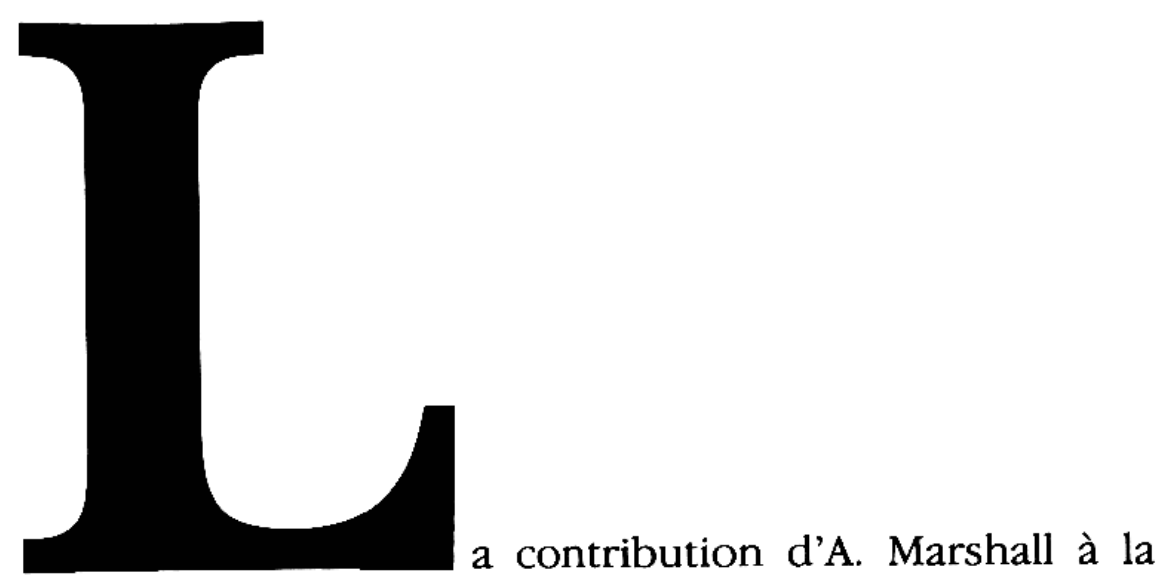

mise en place des fondements théoriques de l'économie industrielle est aujourd'hui assez généralement reconnue par les commentateurs (A. Phillips et R.E. Stevenson [1974], D.A. Morris et D.J. Morris [1979]). Néanmoins, son œuvre a surtout été présentée, dans la littérature anglo-saxonne 
en particulier, comme un "point de départ instrumental (...) une "boîte à outils" qu'il convient d'adapter afin de l'utiliser dans l'étude des comportements des firmes, des structures de marché et des performances industrielles» (A. Maricic [1988], p. 13). Cette interprétation dominante tient pour l'essentiel à la «curieuse négligence» (H.H. Liebhafsky [1955]) d'une partie importante de ses écrits plus spécifiquement consacrés à l'analyse des faits industriels. Mais, paradoxalement, la redécouverte récente, des deux autres grands ouvrages d'A. Marshall, The economics of industry $[1879]^{1}$ et Industry and trade $[1919]^{2}$, ainsi d'ailleurs que la publication de ses écrits de jeunesse ${ }^{3}$, n'ont pas eu pour conséquence de relativiser la place, dans la structure comme dans la portée de son œuvre, des Principles of economics. En autorisant une lecture renouvellée des éditions successives du texte de 1890 , elles ont tout au contraire favorisé l'émergence d'une interprétation plus globale de la pensée marshallienne ${ }^{4}$. Dans un tel contexte, l'intérêt des chapitres de nature, au moins en apparence, plus descriptive qui abondent dans les Principes, ne pouvait plus longtemps échapper aux lecteurs attentifs. Il en est ainsi, par exemple, des 5 chapitres du Livre IV consacrés à l'Organisation industrielle et, notamment, du chapitre $\mathrm{X}$ sur « la concentration d'industries spécialisées dans certaines localités" dont nous proposons ci-dessus une traduction française ${ }^{5}$ et auquel nous consacrons le présent commentaire.

L'analyse que nous propose A. Marshall de la localisation industrielle dans ce court chapitre ${ }^{6}$ du Livre IV des Principes d'économie politique présente ceci d'original qu'elle n'accorde pas de véritable importance à des causes premières exogènes, contrairement à beaucoup de travaux sur le sujet, y compris les plus récents et les plus sophistiqués. Bien sûr, Marshall commence son texte en énumérant ces conditions physiques d'offre ou de de- 
mande qui sont, le plus souvent sinon toujours, à l'origine des localisations industrielles. Mais c'est pour dire tout aussitôt que l'on est en présence de causes essentiellement accidentelles et que si «nous avons vu comment la nature physique agit sur l'énergie de l'homme, comment un climat vivifiant le stimule, et comment l'ouverture de champs nouveaux pour son travail l'encourage à tenter des entreprises risquées (...) nous avons vu aussi que l'usage qu'il fait de ces avantages dépend de ses idéaux de vie, et que, par conséquent, les fils de la religion, de la politique et de l'économie sont inextricablement entrecroisés dans l'histoire du monde» (Principles of economics, 224-25). Dès lors, ce qui lui semble beaucoup plus important, c'est de détecter les avantages internes d'une localisation particulière, c'est-à-dire, ceux qu'elle engendre d'elle-même dans le temps, et de déterminer les conditions de sa viabilité, ces deux questions étant à l'évidence étroitement imbriquées l'une à l'autre : «Aussi, écrit-il, devons-nous, pour le moment, laisser de côté ces vastes mouvements de localisation industrielle, pour nous intéresser au sort des groupes d'ouvriers qualifiés, qui se trouvent rassemblés dans les limites étroites d'une ville manufacturière ou d'un district industriel fortement peuplé» (Principles of economics p. 225).

Ces avantages créés par une localisation particulière sous la forme, soit d'une ville manufacturière, soit d'un district industriel, sont de deux ordres : il y a ceux attachés aux ressources humaines rassemblées et il y a ceux qui viennent de l'interdépendance technique des activités créées. Comme nous allons le voir, il n'y a pas lieu de les dissocier et, d'ailleurs, Marshall les présente dans un seul et même mouvement. S'agissant des ressources humaines, l'avantage tient à la transmission et à l'enrichissement des compétences au cours du temps. Ainsi, "les secrets de l'industrie cessent d'être des secrets; ils sont pour ainsi 
dire dans l'air et les enfants apprennent inconsciemment beaucoup d'entre eux" (Principles of economics p. 225). Il existe ce que Marshall appelle ailleurs (Industry and trade, p. 287) une «atmosphère industrielle» qui favorise la maîtrise et le développement des compétences, et qui est l'expression d'un véritable processus culturel. Un aspect essentiel de l'environnement ainsi construit est la capacité d'innovation qui est avant tout une capacité d'acquisition de nouvelles compétences : «Le travail bien fait est immédiatement reconnu et l'on discute aussitôt les mérites des inventions et des améliorations apportées aux machines, aux procédés et à l'organisation générale de l'industrie; si quelqu'un trouve une idée nouvelle, elle est aussitôt reprise par d'autres et combinée avec des suggestions de leur propre crû; elle devient ainsi la source d'autres idées nouvelles" (Principles of economics, p. 225) ; et d'ajouter immédiatement : «bientôt des industries auxiliaires naissent dans le voisinage, fournissant à l'industrie principale les instruments et les matières premières, organisant son trafic et lui permettant dans bien des cas des économies de matières" (ibid). L'intégration des compétences et des activités (principales et auxiliaires) est présentée comme la principale source de l'innovation et, par suite, du développement. C'est elle qui confère son efficacité dynamique à la ville manufacturière ou au district industriel.

L'autre avantage dynamique, sur lequel Marshall met l'accent, réside dans les relations d'interdépendance proprement techniques. Les industries (auxiliaires) dédiées, elles-mêmes, à un segment étroit du processus de production, mais travaillant pour un grand nombre d'industries voisines, sont en mesure, grâce à une utilisation constante qui en compense la dépense, de détenir des machines hautement spécialisées dont le coût est élevé et le taux d'obsolescence rapide. C'est là l'effet d'économies 
externes techniques (et non nécessairement d'agglomération). Par ailleurs, une communication constante entre les constructeurs et les utilisateurs de machines, qui constitue une forme d'échange et d'enrichissement de compétences, autorise une certaine standardisation et, surtout, certaines spécialisations. C'est là l'effet d'économies externes fortement liées à la localisation. Tout cela concourt à l'efficacité dynamique de la ville ou du district.

Ces avantages créés (ou internes) d'une localisation particulière font que celle-ci est doublement caractérisée par la nature des activités agglomérées et par la configuration du marché du travail qu'elle a engendré. Pour Marshall, certes, le district industriel type est un district textile ou un district métallurgique, par exemple, c'est-àdire qu'il est composé d'entreprises appartenant à la même branche industrielle. Toutefois, celle-ci est définie de manière large puisqu'elle comprend une industrie principale et des industries auxiliaires. Ces dernières comprennent aussi bien des activités situées techniquement en amont ou en aval, qui sont, donc, dans une relation verticale avec l'industrie principale, que des activités complémentaires, surtout des activités de service à l'industrie principale. Ainsi, l'ensemble des activités productives d'un district industriel forme-t-il un seul et unique processus de production complexe dont la dimension essentielle est celle du temps, en l'occurence le temps de l'apprentissage, de l'élaboration et de la mise en ouvre d'une capacité de production spécifique. En bref, comme le souligne G. Becattini ([1989 b], p. 5), «le district est un exemple de réalisation localisée du processus de division du travail, qui n'est ni dilué dans le marché général, ni concentré sur une seule ou sur un petit nombre d'entreprises. La localisation signifie ici quelque chose de différent de la concentration accidentelle en un même lieu de processus de production qui y auraient été attirés par des facteurs de localisation préexistants. Plutôt, 
les entreprises s'enracinent dans le territoire, et ce résultat ne peut être compris indépendamment de son développement historique».

Le district industriel constitue, par ailleurs, un marché efficient du travail : «une industrie localisée tire toujours un grand avantage à constituer un marché permanent pour un même type de qualification» écrit Marshall. "Les employeurs sont tout à fait disposés à s'adresser à un endroit où ils ont toutes chances de trouver un bon choix d'ouvriers dotés des qualifications particulières qu'ils recherchent; de leur côté, les ouvriers cherchant du travail se dirigent naturellement vers les endroits où se trouvent beaucoup d'employeurs ayant besoin de main-d'œuvre de même qualification que la leur et où, par conséquent, ils ont des chances de trouver un marché avantageux» (Principles of economics, pp. 225-26). Un tel marché du travail est efficace, non seulement d'un point de vue statique d'affectation des ressources en travail, mais aussi du point de vue dynamique en permettant, de par son fonctionnement, la création, localement, de nouvelles compétences. Son efficacité vient en partie de ce "qu'il y a souvent des liens étroits entre employeurs et employés» (Principles of economics, p. 226), c'est-à-dire du fait que ce n'est pas un marché pur, mais qu'il présente certains traits de ce qu'on appelle, dans la terminologie moderne, un marché interne, de telle sorte que l'employeur peut, notamment, obtenir une meilleure évaluation des qualités professionnelles de ses employés. Les transactions réalisées sur ce marché le sont entre un nombre assez limité de participants dans le cadre de relations continues, d'où il ressort des mécanismes incitatifs spécifiques dont l'un des résultats est l'enrichissement des compétences.

L'accent mis sur les avantages créés plutôt que sur les avantages innés conduit Marshall à poser le problème de la viabilité de la localisation, conçue comme un proces- 
sus se déroulant dans le temps. En effet, bien qu'il engage l'analyse sur ce point en évoquant les désavantages d'une localisation industrielle particulière, c'est à dire, dans une perspective qui semble statique, il devient rapidement clair que son objet est d'identifier les causes de la croissance continue des industries d'une ville ou d'un district. Ces causes, il les trouve dans le degré de diversité du marché du travail, d'une part, et dans celui des activités localisées, d'autre part.

La diversité du marché du travail permet, selon Marshall, de concilier un coût du travail qui n'est pas excessif et des revenus familiaux suffisants : «Dans les districts sidérurgiques, où il n'y a ni industries textiles, ni aucune autre industrie susceptible de donner du travail aux femmes et aux enfants, les salaires sont hauts et le coût du travail élevé pour l'employeur, bien que les revenus monétaires moyens des familles soient faibles. Mais le remède à ce mal est évident, qui réside dans le développement dans la même région d'industries ayant des caractéristiques complémentaires» (Principles of economics, p. 226).

La diversité des activités permet d'éviter le contrecoup des crises sectorielles : «Un district qui dépend à titre principal d'une seule industrie s'expose à une crise très grave, en cas de diminution de la demande pour ses produits ou de pénurie de l'offre des matières premières qu'il utilise. Ce risque est (...) dans une large mesure évité dans les grandes villes ou les grands districts industriels, où plusieurs industries différentes ont pu se développer puissamment" (Principles of economics, p. 227). Il s'agit, en réalité, de faire en sorte que la disparition de certains avantages innés (exogènes, par conséquent) ne fasse pas perdre, en même temps, le bénéfice des avantages créés. Toutefois, si ces derniers sont réellement importants, alors la diversification des compétences et des activités a atteint un tel degré que la viabilité du processus de localisation 
est assurée : il a pu échapper aux conséquences des aléas frappant les avantages innés.

Ce même problème d'articulation et de confrontation entre avantages innés et avantages créés surgit quand Marshall entreprend, finalement, d'opérer une distinction entre la ville (commerciale) et le district (industriel). Selon lui, la valeur qu'un centre urbain a pour le commerce est plus grande que celle qu'il a pour l'industrie et cela a pour effet d'en exclure les usines. Cette concurrence pour l'occupation de l'espace existe aussi entre les employés du commerce et les travailleurs de l'industrie. La conséquence en est «qu'aujourd'hui, les fabriques se groupent dans les faubourgs des grandes villes et dans les districts industriels limitrophes plutôt qu'au cœur des villes elles-mêmes» (Principles of economics, p. 226). En fait, la séparation entre villes et districts devient un facteur de viabilité du processus de localisation qui les englobe, en conciliant le poids d'avantages innés différenciés et la création d'avantages spécifiques.

L'analyse du phénomène de localisation fait ici partie de l'analyse plus vaste d'un processus de développement au sens schumpeterien du terme.

La distinction effectuée par Marshall entre ce que nous appellerons des avantages généraux et des avantages spécifiques éclaire de manière essentielle la compréhension que l'on doit avoir des phénomènes de localisation industrielle.

En effet, la question cruciale en cette matière est, finalement, celle de la perennité d'une agglomération d'activités productives dans un lieu déterminé et du système d'emploi qui l'accompagne. Or, les avantages dits généraux, souvent d'ordre physique, et en tous cas externes, dans le sens où ils ne dépendent pas de l'action des entreprises, ne sont pas une garantie de perennité. D'une part, de tels avantages ont précisément ce caractère général qui 
veut dire qu'ils sont réunis dans plusieurs lieux concurrents. D'autre part, ils sont intrinsèquement liés à telle ou telle technologie matériellement incarnée (les mines de fer et la sidérurgie par exemple), auquel cas leur intérêt et leur impact disparaissent quand cette technologie devient obsolète. En revanche, les avantages spécifiques, tels qu'ils sont envisagés par Marshall, ont ceci d'essentiel qu'ils sont construits en chemin et porteurs de diversification et de développement au sens schumpeterien du terme, de telle sorte qu'ils assurent la constitution d'un système productif localisé stable quoique évolutif quant à ses caractères matériels.

Certes, avec ce genre de distinction, Marshall ne fait qu'avancer des critères d'évaluation économique de la localisation industrielle. Mais c'est à partir d'eux qu'il est possible d'envisager différents modes de construction des avantages spécifiques suivant les contextes organisationnels, institutionnels, voire culturels. Les districts industriels italiens (tel Prato) sont l'un de ces modes de construction d'avantages spécifiques, caractérisés en l'occurence par un système relationnel particulier au sein de la région concernée, entre les entreprises aussi bien qu'avec des marchés souvent lointains. Mais ils ne sont que l'un de ces modes. Ailleurs, dans d'autres pays, un héritage organisationnel, institutionnel, culturel et technologique différent entraîne des processus de localisation industrielle différents.

Il apparaît à l'analyse que les développements qu'A. Marshall consacre aux formes concrètes d'organisation et de coordination de l'activité économique ont été pour l'essentiel ignorés au profit des seuls phénomènes de marché parce que apparemment trop descriptifs. Ils pourraient le demeurer encore longtemps si l'on persiste à les subordonner à des conceptions étroites de la production et donc, des coûts. et des rendements. Nous savons que Marshall associe la productivité croissante d'une industrie 
à la combinaison de deux types d'économies de production : celles qui sont externes aux firmes individuelles mais internes à la branche en ce qu'elles dépendent du «développement général de l'industrie» et celles qui sont internes aux firmes de la branche et qui découlent de leur efficacité dans la mise en œuvre de leurs capacités organisationnelles et managériales. Les premières, essentiellement rattachées aux phénomènes de localisation industrielle, sont largement illustrées dans le texte et le commentaire qui précèdent. Les secondes proviennent, selon Marshall, de la division du travail et du recours accrû aux machines spécialisées au sein de la firme, qui permettent une mobilisation plus efficace du capital et de la main d'œuvre ${ }^{7}$. Nous savons aussi que c'est précisément sur ces questions qu'ont porté certaines des critiques les plus vives qu'ait eu à subir la théorie marshallienne. Souvenons-nous par exemple des propos de P. Sraffa dans son article de $1925^{8}$, (qui fut, avec celui de $1926^{9}$, particulièrement dévastateur pour l'analyse en termes d'équilibre partiel et auquel on doit sans aucun doute l'origine des travaux sur la concurrence imparfaite), soulignant que : «Dans The economics of industry, qui contient la première expression organique de sa doctrine, Marshall fait dériver directement la loi de la productivité croissante de la "loi de la division du travail" et considère cette dernière comme dépendante au premier chef "de la dimension des usines où le travail est effectué"; or, en supposant parmi les causes de la diminution du coût une circonstance incompatible avec la libre concurrence, il frise l'erreur qu'il devait par la suite lui-même dénoncer» ${ }^{10}$. Or, comme le fait remarquer $G$. Becattini ${ }^{11}$, la critique de Sraffa tend à occulter les réserves très tôt exprimées par Marshall quant à la conception courante de la division du travail, qui lierait l'obtention quasi exclusive de ses avantages les plus importants à «la concentration de grandes masses de travailleurs dans des vastes 
établissements ${ }^{12}$. Dans The pure theory of domestic values, dont Sraffa n'a sans doute pas pu avoir connaissance, Marshall affirme de manière fort explicite, que la plupart des avantages de la division du travail peuvent aussi être obtenus dans les secteurs d'activité composés d'un grand nombre de firmes de petite taille : «on peut par conséquent conclure qu'un accroissement de la quantité totale d'un bien manufacturé ne peut en général manquer d'occasionner des économies accrues dans la production, que la charge de cette production soit répartie sur un grand nombre de petits capitalistes, ou qu'elle soit concentrée dans les mains d'un nombre relativement restreint de grandes firmes ${ }^{13}$.

Que signifie ce débat, sinon que la production ne peut pas être ramenée à un problème d'affectation des ressources (ce qu'implique la référence à une fonction de production et au postulat d'efficacité technologique que celle-ci véhicule) et qu'elle doit être vue fondamentalement comme un processus de création, ce dont Kaldor [1972] a eu l'intuition. Si l'on admet cela, il est alors possible d'envisager différentes formes d'organisation industrielle ayant leur efficacité propre. Une grande firme ou une agglomération de petites firmes, différentes formes de territorialisation de l'activité productive ont chacune leur logique de développement propre et donc leurs conditions de viabilité propres. Nul critère ne permet d'avancer a priori la supériorité d'une forme d'organisation sur l'autre. Ceci plaide en faveur d'une diversité acceptée, à condition qu'elle soit maîtrisée. Tel est sans doute l'enjeu auquel doit faire face la Communauté économique européenne dans la mise en œuvre de la politique industrielle, de la politique de la recherche, de la politique régionale avec l'ouverture du grand marché. 


\section{Notes}

1. Cet ouvrage a été écrit en collaboration avec Mary Paley Marshall.

2. Une traduction française en a été proposée en 1934 par G. Leduc sous le titre L'industrie et le commerce (Editions Marcel Giard, Paris, 2 volumes). Sur l'importance de ce texte, voir par exemple P.L. Williams [1986].

3. Cf. J.K. Whitaker ed. [1975] et M. Dardi [1984].

4. Cf. notamment P.W.S Andrews [1951] et G. Becattini [1975]. C'est en outre à ce dernier auteur que l'on doit la toute première caractérisation du concept de "district industriel marshallien" (G. Becattini, ed. [1987] et G. Becattini [1989a] et [1989b].

5. La seule traduction française disponible des Principles of economics est celle de la quatrième édition [1898] par F. Sauvaire Jourdan. Publiée en 1906-1909 (Editions Giard et Brière, Paris), elle a été réimprimée en 1971 par les éditions Gordon and Breach (Paris et Londres). La traduction que nous proposons ici a été effectuée sur la base de la huitième édition d'Octobre 1920 et tient évidemment compte des modifications introduites par A. Marshall au cours des éditions successives.

6. La question de la localisation industrielle est également évoquée, quoique de façon moins systématique, dans The economics of industry (Livre II, Chapitre VII) et dans Industry and trade (Livre II, Chapitre 6, pp. 439-449 de l'édition française). En fait, les fondements du concept marshallien d'«industrie localisée» ou «district industriel "sont déjà parfaitement en place dès les tout premiers écrits d'A.
Marshall, et notamment dans un petit opuscule rédigé vers 1875: The pure theory of foreign trade, the pure theory of domestic values (Cf. J.K. Whitaker ed. [1975], en particulier, Vol. 2, pp. 195-198).

7. La baisse du coût moyen ainsi obtenue lorsque la production s'accroit, peut toutefois être contrecarrée, à partir d'un certain seuil, par l'apparition de "déséconomies internes " liées à des difficultés de gestion ou de commercialisation. Où l'on retrouve l'explication bien connue de la forme en U de la courbe de coût moyen de longue période.

8. Sur les relations entre côेt et quantité produite, in Sraffa [1975], p. 1-49.

9. Les lois des rendements en régime de concurrence, in Sraffa [1975], p. $51-68$.

10. Sraffa [1975], p. 28 . Sraffa concluait : « En définitive, (...) il ne peut pas exister, dans un système statique de libre concurrence et dans la détermination des équilibres partiels, de courbes de coûts non proportionnels (sinon en des cas exceptionnels), sans qu'avec elles soient introduites des hypothèses contredisant la nature du système. (...) De ce point de vue, (...) nous devons donc admettre que les marchandises sont généralement produites à coûts constants ", ibid, p. $48-49$.

11. G. Becattini [1975], p. xcviii. Ce dernier y signale une autre dimension importante de la division du travail entrevue par Marshall mais occultée sous l'effet de la critique sraffaienne des rendements croissants. Il s'agit du rôle 
de la demande ou de l'«étendue du marché" pour reprendre l'expression smithienne - dont l'augmentation favorise «une subdivision interne croissante de l'industrie». Cette idée sera reprise et développée par A. A. Young
[1928], article traduit et commenté dans : J. De Bandt, J-L Ravix et P-N. Romani [1990].

12. Cf. Whitaker [1975], p. 195.

13. ibid, p. 198. 


\section{Références}

P.W.S. Andrews [1951] : Industrial analysis in economics, in T. Wilson \& P.W.S. Andrews, Oxford studies in price mechanism, Oxford University Press, pp. 139-172.

R. Arena, L. Benzoni, J. De Bandt, \& PM Romani eds [1988] : Traité d'économie industrielle, Economica, $\mathrm{Pa}$ ris.

G. Becattini [1975] : Invita a una rilletura di Marsball - Introduction à A. Marshall et M.P. Marshall, Economia della produzione, ISEDI, Milan (Trad. italienne de The economics of industry [1879]).

G. Becattini ed. [1987] ; Mercato e forze locali : il distretto industriale, Il Mulino, Bologne.

G. Becattini [1989.a] : Sectors and/or districts: some remarks on the conceptual foundations of industrial economics, in E. Goodman \& J. Bamford eds, Small firms and industrial districts in Italy, Routledge, London, pp. 123-135.

G. Becattini [1989.b] : Some thoughts on the Marshallian industrial district as a socio economic notion, Communication à l'école d'été méditerranéenne d'économie industrielle, $4^{\mathrm{e}}$ session, Institut d'études scientifiques de Cargèse, Septembre, Miméo.

M. Dardi [1984] : Il giovane Marshall, Il Mulino, Bologne.

J. De Bandt, J.L. Ravix \& P-M Romani [1990] : Allyn Young: une approche de la dynamique industrielle, Revue française d'économie, $\mathrm{Vol} . \mathrm{V}, \mathrm{n}^{\circ} 2$, pp. 111-138.

D.A. Hay \& D.J. Morris [1979] : Indus- trial economics. Theory and evidence, Oxford University Press, Oxford.

N. Kaldor [1972] : The irrelevance of equilibrium economics, The economic journal, 82, Décembre, pp. 12371255 (Traduction française dans $\mathrm{R}$. Boyer et alii [1987], Economie et instabilité : Nicholas Kaldor, Economica, Paris.

H.H. Liebhafsky [1955] : A curious case of neglect: Marshall's industry and trade, Canadian journal of economic and political science, Vol. XXI, n³, Août, pp. 339-353.

A. Maricic [1988] : Les fondements marshalliens de l'économie industrielle, in R. Arena, L. Benzoni, J. De Bandt \& P-M Romani eds [1988], pp. 13-21.

A. Marshall \& M. Paley Marshall [1879] : The economics of industry, Macmillan, Londres.

A. Marshall [1906] : Principes d'économie politique, trad. française de la $4^{*}$ édition [1898], Ed. Giard et Brière, Paris, réimpression de 1971, Ed. Gordon $\&$ Breach, Paris, 2 volumes.

A. Marshall [1920] : Principles of economics, $8^{e}$ édition, réimpression de 1979, The Macmillan Press Ltd, London.

A. Marshall [1934] : L'industrie et le commerce, trad. française de Industry and trade [1919], Ed. Marcel Giard, Paris, 2 volumes.

A. Phillips \& R.E. Stevenson [1974] : The bistorical development of industrial organization, History of poli- 
Jean-luc Gaffard / Paul-Marie Romani 185

tical economy, Vol. $6, \mathrm{n}^{\circ} 3$, pp. 324- dustry and trade in the analysis of 342 .

P. Sraffa [1975] : Ecrits d'économie politique, Economica, Paris.

Alfred Marsball, in $\mathrm{K}$. Tucker \& $\mathrm{C}$. Baden-Fuller eds, Firms and markets. Croom Helm, London, pp. 227-256.

J.K. Whitaker ed. [1975] : The early writings of Alfred Marshall, 1867. 1890, The Macmillan Press Ltd, London, 2 volumes.

A.A. Young [1928] : Increasing returns and economics progress, The economic journal, Vol. 38 , pp. $527-542$ (trad. française dans J. De Bandt, J.L. P.L. Williams [1986] : The place of inRavix \& P-M Romani [1990]). 